\title{
Immunodysfunction in Acute-on-Chronic Liver Failure
}

\author{
Christian M. Lange ${ }^{a} \quad$ Richard Moreau ${ }^{b}$ \\ a Medizinische Klinik 1, Klinikum der Johann Wolfgang Goethe-Universität, Frankfurt/Main, Germany; \\ ${ }^{b}$ Centre de Recherche sur I'Inflammation (CRI), Institut National de la Santé et de la Recherche Médicale (INSERM), \\ Université Paris-Diderot, Paris, France
}

\section{Keywords \\ Liver cirrhosis - Systemic inflammation - Interleukin-22 . Granulocyte colony-stimulating factor, G-CSF . \\ Bacterial translocation}

\section{Summary}

Background: Increasing evidence reveals a close and reciprocal link between acute-on-chronic liver failure (ACLF) and immunodysfunction. Methods: A literature search in PubMed and abstract databases of relevant congresses was performed. Results: Important characteristics of liver cirrhosis like tissue hypoxia, cell death, or bacterial translocation maintain a state of chronic inflammation. Precipitating events of ACLF such as infections or alcoholic hepatitis are capable of strongly augmenting cirrhosis-associated systemic inflammation to grades sufficient to induce ACLF-defining organ failures. Chronic systemic inflammation, however, is causally linked to profound immunosuppression. As a consequence, patients with liver cirrhosis and in particular with ACLF are at high risk for severe infections. Promising strategies to ameliorate immunodysfunction, like albumin substitution, administration of recombinant interleukin-22 or granulocyte colony-stimulating factor, antibiotic prophylaxis, or anticoagulation, are under development and offer the chance to specifically prevent and treat ACLF. Conclusion: A better understanding of the immunopathology of ACLF will likely translate into the implementation of specific therapeutic modalities to prevent and overcome ACLF.

\section{Pathophysiology and Phenotypes of \\ Immunodysfunction in Liver Cirrhosis and \\ Acute-on-Chronic Liver Failure}

\section{Liver Cirrhosis: A Chronic Inflammatory Disease}

The development of liver fibrosis and cirrhosis is a relatively uniform response to heterogeneous drivers of chronic hepatic inflammation such as chronic viral hepatitis, excessive alcohol exposure, metabolic stress, or autoimmune responses against liver antigens [1]. Key elements of liver fibrogenesis include activation of hepatic stellate cells, matrix deposition and scarring, neoangiogenesis, and activation of local immune cells like macrophages [1]. These processes result in progressive portal hypertension, stress and death of parenchymal and non-parenchymal liver cells, production of chemokines, cytokines, growth factors, reactive oxygen species (ROS), and activation of local and further recruitment of circulating immune cells, such as Ly-6C+ macrophages $[1,2]$. The production of such inflammatory mediators and - importantly the release of so-called damage-associated molecular patterns (DAMPs), such as high-mobility group protein B1 (HMGB1), histones, ATP, or urate from dying cells, are capable of transforming local hepatic inflammation to a systemic inflammatory response. This is characterized by increased serum levels of inflammatory mediators and activation of innate and adaptive immune cells in the circulation, in lymphoid organs, and in extrahepatic tissues [3]. Hence, liver cirrhosis is not only a result of dysregulated hepatic immune responses but also a driver of local and systemic inflammation, which is further amplified by cardinal features of liver cirrhosis, namely portal hypertension, tissue hypoxia in the intestines, liver or kidneys, intestinal dysbiosis, and bacterial translocation.

Besides the liver, the intestines play a key role in the pathogenesis of cirrhosis-associated inflammation. Portal hypertension leads to an intestinal edema characterized by tissue hypoxia and dilatation of the intercellular epithelial spaces [4]. Furthermore, liver cirrhosis is associated with local intestinal inflammation as well as suppression of important mediators of mucosal defense against

\section{KARGER}

(๑) 2018 S. Karger GmbH, Freiburg

Fax +497614520714
Prof. Dr. med. Christian M. Lange Department of Gastroenterology and Hepatology University Hospital Essen

Hufelandstraße 55, 45147 Essen, Germany Christian.lange@uk-essen.de 
pathogens. Both in humans with liver cirrhosis and in animal models, increased expression of proinflammatory cytokines, such as tumor necrosis factor(TNF)- $\alpha$, interferon(IFN)- $\gamma$, or interleukin(IL)-1, as well as increased frequencies and activation status of immune cells in the intestinal wall have been identified [5]. Inflammatory mediators such as TNF- $\alpha$ directly affect the integrity of the intestinal barrier by downregulating tight junction proteins and antimicrobial lectins like antimicrobial-regenerating islet-derived protein 3 gamma [6]. Hence, liver-cirrhosis intestinal inflammation, together with portal hypertension, increases the intestinal permeability as a prerequisite for efficient intestinal bacterial translocation. Intestinal dysbiosis is another important feature of liver cirrhosis and acute-on-chronic liver failure (ACLF) [7, 8]. Patients with liver cirrhosis harbor a less diverse microbiome than healthy individuals, the composition of the intestinal microbiome is shifted from beneficial (e.g. Bifidobacterium spp.) to potentially pathogenic (e.g. Enterococcus spp. or Veillonellaceae spp.) species, and the upper gastrointestinal tract harbors higher quantities of bacteria (i.e. small intestinal bacterial overgrowth) compared to healthy individuals $[5,7,8]$. Cirrhosis-associated intestinal dysbiosis is a consequence of a variety of factors including impaired intestinal motility, a dysregulated mucosal immune system with reduced secretion of antimicrobial proteins, low gastric acidity (in particular in patients on proton pump inhibitors), and altered composition of bile salts reaching the intestines [5].

As a consequence of intestinal dysbiosis and increased intestinal permeability, intestinal translocation of bacteria and of bacterial products (so-called pathogen-associated molecular patterns (PAMPs)) like lipopolysaccharide (LPS), flagellin, or peptidoglycans to the mesenteric lymph nodes as well as portal and systemic circulation occurs [9]. Bacterial translocation increases with severity of liver cirrhosis and is highest in patients with ACLF [10]. Bacterial translocation further exacerbates hepatic and systemic inflammation by stimulation of immune cells in the circulation, in lymphatic tissues, and in the liver; however, it also represents a source of life-threatening infections such as spontaneous bacterial peritonitis (SBP) [9]. Beyond the key phenomenon of bacterial translocation, intestinal dysbiosis is also associated with an altered bacterial metabolome, which can be protective (e.g. production of butyrate by Ruminococcaceae) but also pathogenic, e.g. by contributing to the development of hepatic encephalopathy, although additional research is needed to fully understand the impact of the bacterial metabolome in liver cirrhosis and ACLF [7, 8]. Of note, intestinal dysbiosis, inflammation and permeability are particularly pronounced in patients with ongoing alcoholism, because ethanol and its metabolite acetaldehyde strongly impact on mucosal immunology and the intestinal microbiome [11].

Exacerbation of Systemic Inflammation during Progression to ACLF

The above-described mechanisms lead to a chronic, low-grade systemic inflammation already in patients with compensated liver cirrhosis. Patients with acute decompensation of liver cirrhosis show significantly higher grades of inflammation; however, the highest levels of systemic inflammation were consistently observed in patients with ACLF $[12,13]$. ACLF can be triggered by partially well-defined precipitating events such as infections, alcoholic hepatitis, reactivation of hepatitis $\mathrm{B}$, superinfections with hepatitis $\mathrm{E}$ virus, or hepatotoxic drugs $[13,14]$. These precipitating events are capable of strongly augmenting cirrhosis-associated systemic inflammation, which may result in an overwhelming production of ROS, cytokines, chemokines, growth factors, proteases, eicosanoids, and activation of innate and adaptive immune cells. The degree of systemic inflammation is reflected by an increase of white blood cell (WBC) count and C-reactive protein (CRP), which predict progression of acute decompensation to ACLF and mortality from ACLF, as well as by a number of proinflammatory cytokines such as TNF- $\alpha$, IL-6, or IL-8 [12]. However, it is important to note that the serum concentrations of some other proinflammatory cytokines like IFN- $\alpha$, IFN- $\gamma$, IL-17A, or IL-7 were higher in patients with decompensated cirrhosis compared to healthy controls but that no further increase has been observed in patients with ACLF or with progression to ACLF [12]. These findings indicate that a more comprehensive description of inflammatory phenotypes in patients with or without ACLF may be warranted.

\section{Immunosuppression Coexists with Systemic Inflammation}

As described above, augmented systemic inflammation is strongly associated with ACLF. However, it is highly relevant to acknowledge that liver cirrhosis is not only associated with systemic inflammation but also with profound immunosuppression. In parallel to an increased production of proinflammatory cytokines like IL-6 or TNF- $\alpha$, serum concentrations of anti-inflammatory cytokines like IL-10 or IL-1ra are progressively increasing from compensated to decompensated cirrhosis, in particular with progression to ACLF [12]. In addition, liver cirrhosis is associated with profound alterations of cellular immune responses. For example, monocytes of patients with liver cirrhosis are increased in frequency and activated phenotype, but they do not sufficiently respond to further stimulation with LPS, a phenomenon called LPS tolerance [3]. In patients with ACLF, there is high frequency of peripheral blood monocytes expressing the tyrosine-protein kinase Mer (encoded by MERTK), which suppresses antibacterial monocyte functions [15]. These regulatory monocytes occur in parallel to the storm of proinflammatory cytokines, indicating that the profound inflammation of ACLF does not result in effective antibacterial immune responses [15]. Relevant changes in the adaptive immune cell compartment have been described as well. Patients with liver cirrhosis have lower frequencies of naïve CD4+ and CD8+ T cells and of memory B cells, whereas frequencies of activated $\mathrm{T}$ cells with inappropriate functional properties are increased [3]. Yet, a more detailed understanding of adaptive immunity in ACLF is warranted. Finally, liver cirrhosis is associated with a reduced capacity of the liver to exert its proprietary immune functions, including clearance of intestinal and systemic pathogens, antigen presentation, or production of acute-phase and complement proteins [16].

In principle, upregulation of anti-inflammatory cytokines, inhibitory molecules like tyrosine-protein kinase Mer and other im- 


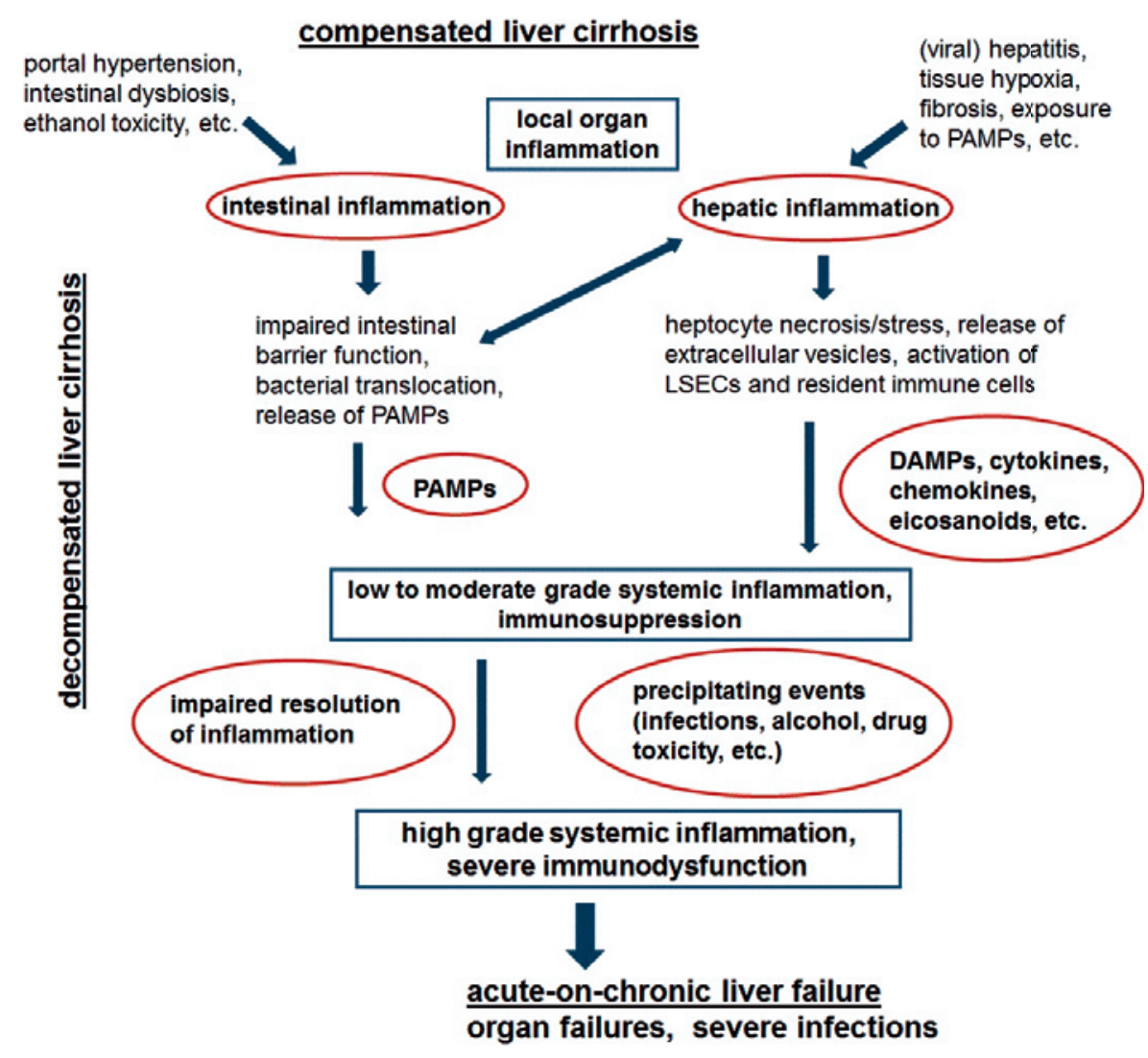

mune checkpoints, and expansion of immunoregulatory cell populations is an inclusive feature of normal immune responses aiming to limit collateral damage of inflammation, an adequate resolution of inflammation, and tissue repair [17]. Failure of adequate resolution of inflammation, partially due to continuous exposure to DAMPs and PAMPs in liver cirrhosis and ACLF, therefore ultimately results in immunodysfunction, i.e., a parallel prolonged state of systemic inflammation and immunosuppression (fig. 1).

\section{Clinical Consequences of Immunodysfunction}

\section{Inflammation as a Driver of Organ Failures}

Already low-grade systemic inflammation in compensated or stable decompensated liver cirrhosis is considered to cause or augment relevant clinical signs and symptoms like hyperdynamic circulation, fatigue, or (minimal) hepatic encephalopathy (HE). In rat models of liver cirrhosis, endotoxin-induced TNF- $\alpha$ reduces cardiac contractility and increases arterial vasodilatation by inducing nitric oxide production, which can be ameliorated by selective bowel decontamination [18, 19]. Higher grades of systemic inflammation in ACLF are strongly associated with - and likely causally linked to - organ failures, which constitute the discriminative characteristic of ACLF versus acutely decompensated cirrhosis [12]. For example, clinical association studies have shown that TNF- $\alpha$ serum levels correlate with progression to and severity of $\mathrm{HE}$ in patients with liver failure, and microglia activation is a typical finding in HE $[20,21]$. In animal models of liver cirrhosis, cerebral recruitment of monocytes, which produce TNF- $\alpha$, as well as endotoxemia-induced brain edema and inflammation have been documented [20, 22]. Increasing evidence suggests that systemic inflammation also promotes renal failure; this is an important notion because the kidneys appear to be particularly sensitive for the systemic alterations associated with advanced liver cirrhosis. Hepatorenal syndrome (HRS) has been considered to be consequence of circulation alterations, i.e. peripheral arterial vasodilatation and impaired cardiac output. However, recent studies suggest that systemic inflammation does not only promote renal failure via impacting on hemodynamics but also by direct effects of inflammatory mediators and PAMPs on renal parenchymal and non-parenchymal cells [23]. Serum and ascites concentrations of IL- 6 and TNF- $\alpha$ are highly predictive for the development of HRS in patients with spontaneous bacterial peritonitis [24]. In sepsis, the concept that PAMPs and DAMPs activate tubular cells and non-parenchymal kidney cells, resulting in renal failure in the absence of sufficient hemodynamic alterations, is already well-established [25]. Research in the field of sepsis and trauma also clearly shows that high-grade inflammation promotes severe organ failures like respiratory failure, kidney failure, or intestinal failure by activation of the coagulation cascade with formation of microthrombi, capillary leakage and micro-vasodilatation, endothelial dysfunction, and mitochondrial dysfunction. Altogether, this results in a deprivation of tissues from oxygen and energy and subsequent cell death [25]. 


\section{Immunosuppression and Risk of Infections}

Due to the above-described features of profound immunosuppression, patients with liver cirrhosis and in particular patients with ACLF are at high risk of developing infections as well as adverse outcomes of infections [26, 27]. In the CANONIC study, $37.3 \%$ of patients with ACLF (vs. 25.1\% without ACLF) had infections at baseline, including SBP in $9.8 \%$, urinary tract infections in $6 \%$, pneumonia in $7.7 \%$, skin and soft tissue infections in $2.9 \%$, and Candida infections in 3.9\% of cases [26]. Patients with ACLF without infection at baseline developed infections during follow-up in $46 \%$ of cases, compared to $18 \%$ of patients with decompensated cirrhosis. Furthermore, infections are a frequent precipitating event of ACLF [26]. Hence, infections are both causes and complications of ACLF. A more detailed description on the management of infections in ACLF is described by Engelmann and Berg [28] in this issue.

\section{Biomarkers of Immunodysfunction}

There is a strong, progressive increase of IL- 6 and IL-10 serum concentrations according to the stage of liver cirrhosis, namely from compensated to decompensated liver cirrhosis and - most intensively - to ACLF. The predictive value of IL-6 appears to be higher than that of CRP or WBC [29]. However, it is important to note that IL-6 serum concentrations in acutely decompensated liver cirrhosis and in ACLF correlate very well with serum concentrations of IL-10 [12]. Hence, increased IL-6 serum levels do not purely reflect a proinflammatory state but also indirectly the above-described immunosuppressive phenotype of liver cirrhosis. Macrophage activation markers, i.e. soluble CD163 and soluble mannose receptor, could be useful diagnostic parameters as they are strongly associated and accurately predict development, grade, and poor outcome of ACLF [30], as well as other complications of liver cirrhosis such as variceal bleeding [31]. In addition, tyrosine-protein kinase Mer expression on monocytes might be considered as a marker of the immunosuppressive phenotype of ACLF [15]. Furthermore, cell death markers such as caspase-cleaved keratin 18, keratin 18, and M30- or M65antigen are (causally) associated with systemic inflammation, and appear to add prognostic information on the risk of progression from acute decompensation to ACLF [32].

In addition to well-performing clinical scores such as CLIF-C scores, the above-described biomarkers may be of value to more accurately predict development and severity of ACLF. However, a major medical need would be a biomarker allowing reliable discrimination of sterile systemic inflammation from infections or sepsis in patients with liver cirrhosis, which can be highly challenging or impossible. As a consequence, empirical administration of broad-spectrum antibiotics is often performed in clinical practice, which bears a substantial risk of drug toxicity, of selecting drugresistant organisms, or of promoting fungal infections. CRP and blood leukocytes are not of value to discriminate infections from sterile inflammation in liver cirrhosis/ACLF. Serum cytokine patterns show a distinct pattern for alcoholic and infection-triggered
ACLF, but these differences are not sufficient for diagnostic purposes due to a significant overlap [12]. In contrast, procalcitonin may help to guide clinical decision making in certain cases. For example, a study in patients with severe alcoholic hepatitis has shown that procalcitonin serum concentrations above $0.45 \mathrm{ng} / \mathrm{ml}$ are frequently associated with the presence of infections (83.3\%) [33]. However, a relevant proportion of $29 \%$ of patients with procalcitonin concentrations below $0.45 \mathrm{ng} / \mathrm{ml}$ had infections in this study, showing that the identification of additional, more reliable biomarkers of infections in patients with liver cirrhosis is warranted.

\section{Therapeutic Strategies to Target \\ Immunodysfunction for the Treatment or Prevention of ACLF}

As described above, there is a strong association between immunodysfunction, i.e. systemic inflammation and immunosuppression, and ACLF. These data from clinical association studies, animal models of liver cirrhosis (no animal model of ACLF is available), and extensive research in the field of sepsis, which shares pathophysiological features with ACLF, suggest a causal relationship between immunodysfunction and development of organ failures, i.e. ACLF. With respect to the development of specific therapies to prevent and treat ACLF, it is important, however, to keep in mind the hypothetical nature of this assumption. Indeed, treatment of systemic inflammation of severe alcoholic hepatitis with glucocorticoids only results in a marginal survival benefit and is associated with an increased risk of subsequent infections [34]. Furthermore, trials have failed to show a benefit of anti-TNF- $\alpha$ therapy in alcoholic hepatitis, which might be explained by an increased risk of infection or by a negative impact on liver regeneration (TNF- $\alpha$ and IL-6 are mandatory for liver regeneration in several models) [35]. Nevertheless, a number of interesting therapeutic strategies are under investigation, as described in the following and in table 1.

\section{Prophylactic Antibiotics}

Primary prophylaxis in patients at higher risk and secondary antibiotic prophylaxis of SBP are well-established measures which significantly reduce the risk of SBP and subsequent complications, including - plausibly - ACLF and - eventually - death. Preliminary and partially conflicting data suggest that rifaximin may not only reduce the incidence of $\mathrm{HE}$ but also improve hemodynamics, systemic inflammation, and complications like HRS in patients with advanced liver cirrhosis [36]. Recently, a large $(n=291)$ randomized study compared prophylactic norfloxacin (400 mg daily) versus placebo for 6 months in patients with Child-Pugh class $\mathrm{C}$ cirrhosis [37]. Preliminary results show that mortality at 6 months was higher in the placebo group (24.5\%) than in the norfloxacin group (15.3\%). Furthermore, the rate of infections was reduced by norfloxacin prophylaxis (20.8 vs. $31.3 \%$ ). Hence, it is likely that the concept of antibiotic prophylaxis in patients with liver cirrhosis will be extended beyond SBP in order to reduce infections and subsequent development of ACLF. 
Table 1. Therapeutic strategies that target immunodysfunction in acute-on-chronic liver failure

\begin{tabular}{|c|c|c|}
\hline & Postulated mechanisms & Evidence \\
\hline $\begin{array}{l}\text { Non-selective } \\
\text { beta-blockers } \\
\text { [47] }\end{array}$ & $\begin{array}{l}\text { improvement of hemodynamics, } \\
\text { reduction of bacterial translocation }\end{array}$ & $\begin{array}{l}\text { high, but uncertainties with respect to } \\
\text { adverse effects in specific patient groups } \\
\text { (details in [51]) }\end{array}$ \\
\hline IL-22 & $\begin{array}{l}\text { improvement of intestinal barriers, } \\
\text { supports liver regeneration }\end{array}$ & proof-of-principle trials are underway \\
\hline G-CSF & $\begin{array}{l}\text { improvement of liver regeneration and } \\
\text { immunodysfunction }\end{array}$ & $\begin{array}{l}\text { survival benefit in smaller trials, large } \\
\text { confirmatory trials are ongoing }\end{array}$ \\
\hline $\begin{array}{l}\text { Anticoagulation } \\
\text { (enoxaparin } \\
\text { and others) [48] }\end{array}$ & $\begin{array}{l}\text { improvement of microcirculation in } \\
\text { intestines and liver, reduction of } \\
\text { bacterial translocation }\end{array}$ & $\begin{array}{l}\text { promising data which require confirma- } \\
\text { tion, additional trials are underway } \\
\text { (details in [52]) }\end{array}$ \\
\hline $\begin{array}{l}\text { Antibiotic } \\
\text { prophylaxis }\end{array}$ & $\begin{array}{l}\text { reduction of bacterial translocation, } \\
\text { prevention of infection, possibly direct } \\
\text { inhibition of inflammation }\end{array}$ & $\begin{array}{l}\text { high, but selection of drug-resistant } \\
\text { organisms may occur }\end{array}$ \\
\hline $\begin{array}{l}\text { Obeticholic acid } \\
\text { [49] }\end{array}$ & $\begin{array}{l}\text { improvement of intestinal dysbiosis, } \\
\text { inflammation, and barrier function }\end{array}$ & $\begin{array}{l}\text { mainly from animal models; currently, } \\
\text { safety concerns in patients with decom- } \\
\text { pensated cirrhosis are examined }\end{array}$ \\
\hline Albumin & $\begin{array}{l}\text { improvement of hemodynamics, } \\
\text { immune modulation }\end{array}$ & $\begin{array}{l}\text { high for the prevention of HRS and } \\
\text { infections }\end{array}$ \\
\hline $\begin{array}{l}\text { Plasma exchange } \\
\qquad[50]\end{array}$ & $\begin{array}{l}\text { removal of PAMPs, DAMPs, cytokines, } \\
\text { etc. from the circulation }\end{array}$ & $\begin{array}{l}\text { preliminary and heterogeneous studies, } \\
\text { further confirmation of survival benefit } \\
\text { required }\end{array}$ \\
\hline
\end{tabular}

$\mathrm{IL}=$ Interleukin; G-CSF = granulocyte colony-stimulating factor; DAMPs = damage-associated molecular patterns; PAMPs = pathogen-associated molecular patterns; HRS = hepatorenal syndrome.

\section{G-CSF}

In animal models, granulocyte-colony stimulating factor (G$\mathrm{CSF}$ ) improves liver regeneration via mobilization of bone marrow-derived stem cells and expansion of hepatic progenitor cells, and ameliorates immune function of leukocytes. Preliminary data suggest that G-CSF therapy might improve outcomes of ACLF. A randomized trial assessed repetitive subcutaneous injections of 5 $\mu \mathrm{g} / \mathrm{kg}$ G-CSF for 1 month $(\mathrm{n}=23)$ or placebo $(\mathrm{n}=24)$ in patients with ACLF [38]. Survival was significantly higher in the G-CSF group (66 vs. $26 \%$ ). Another randomized trial in patients with ACLF and chronic hepatitis B achieved comparable results (48 vs. $21.4 \%$ survival at day 90) [39]. Currently, a large German trial evaluating G-CSF in ACLF is ongoing (Graft study).

\section{Human Albumin}

Human albumin is an established agent for the treatment of HRS as well as for the prevention of HRS in patients requiring large-volume paracentesis or in those with SBP. Beyond its oncotic properties, which improve hemodynamics, beneficial immunemodulatory effects of albumin are increasingly being recognized [40]. Albumin has a remarkable capacity of binding inflammatory mediators such as lipid mediators (prostaglandin E2 is of particular importance), ROS, PAMPs and DAMPs, or cytokines [40, 41]. In addition, albumin may modulate immune reactions by directly acting on endothelial and immune cells. Hence, albumin is currently being investigated in settings beyond the above-mentioned indications. The Italian ANSWER trial assessed weekly administration of albumin plus diuretics for 18 months compared to diuretics alone in patients with liver cirrhosis and ascites. In the albumin group, significantly lower rates of death, paracentesis, refractory ascites, SBP and other infections, or renal failure were observed [42]. In contrast, albumin substitution in patients with liver cirrhosis and other infections than SBP did not improve outcome [43, 44]. Additional trials assessing the impact of albumin on nosocomial infections and the development of ACLF are underway [40].

\section{Interleukin-22}

IL-22 is a member of the IL-10 cytokine family acting on a transmembrane receptor complex consisting of the two subunits IL-22R1 and IL-10R2, which are only expressed on non-immune cells such as hepatocytes, kidney cells, keratinocytes, or epithelial cells of the intestinal or respiratory tract [45]. Of note, IL-22 signaling can be prevented by the soluble IL-22 binding protein (IL22BP) [46]. IL-22 signaling is considered to be protective in chronic liver disease, as it strengthens the intestinal cellular barrier, promotes intestinal immune responses via induction of antimicrobial proteins, and promotes liver regeneration in vitro as well as in animal models [45]. Consequently, intravenous IL-22 is currently being evaluated in a clinical trial in patients with alcoholic hepatitis (NCT02655510). However, it is important to note that high serum 
levels of IL-22 have been associated with adverse outcomes in patients with alcoholic liver disease and ACLF. We have observed that the increase of IL-22 serum levels in ACLF is, however, accompanied by an excessive secretion of IL-22BP (unpublished data). Furthermore, we have noticed that physiological concentrations of IL-22BP observed in ACLF are capable of almost completely inhibiting hepatocellular IL-22 signaling. Hence, high doses of IL-22 could be required for a therapeutic use of IL-22 in patients with ACLF. Furthermore, IL-22 also has proinflammatory functions that may adversely impact on the outcome of patients with severe liver diseases, and a recent study has revealed a protective role of IL-22BP in a murine model of acute liver failure [46]. Nevertheless, IL-22 appears to be a promising candidate to be investigated for the prevention and treatment of ACLF.
ACLF. Organ failures and infections are a cause and a consequence of systemic inflammation and immunosuppression, indicating a reciprocal causal relationship between immunodysfunction and ACLF. Emerging therapies that specifically target immunodysfunction to treat and to prevent ACLF are encouraging.

\section{Acknowledgment}

CML is supported by the Deutsche Forschungsgemeinschaft (LA 2806/2-1 and LA 2806/5-1 to CML).

\section{Disclosure Statement}

CML and RM declare that no conflicts of interest do exist.

\section{Conclusion}

Immunodysfunction, in terms of a parallel existence of systemic inflammation and profound immunosuppression, is a hallmark of

\section{References}

1 Pellicoro A, Ramachandran P, Iredale JP, et al: Liver fibrosis and repair: immune regulation of wound healing in a solid organ. Nat Rev Immunol 2014;14:181194.

2 Tacke F, Zimmermann HW: Macrophage heterogeneity in liver injury and fibrosis. J Hepatol 2014;60:10901096.

3 Albillos A, Lario M, Alvarez-Mon M: Cirrhosis-associated immune dysfunction: distinctive features and clinical relevance. J Hepatol 2014;61:1385-1396.

4 Such J, Guardiola JV, de Juan J, et al: Ultrastructural characteristics of distal duodenum mucosa in patients with cirrhosis. Eur J Gastroenterol Hepatol 2002;14: 371-376.

5 Schnabl B, Brenner DA: Interactions between the in testinal microbiome and liver diseases. Gastroenterology 2014;146:1513-1524.

6 Wang L, Fouts DE, Starkel P, et al: Intestinal REG3 lectins protect against alcoholic steatohepatitis by reducing mucosa-associated microbiota and preventing bacterial translocation. Cell Host Microbe 2016;19:227239.

7 Qin N, Yang F, Li A, et al: Alterations of the human gut microbiome in liver cirrhosis. Nature 2014;513: 59-64.

8 Chen Y, Guo J, Qian G, et al: Gut dysbiosis in acuteon-chronic liver failure and its predictive value for mortality. J Gastroenterol Hepatol 2015;30:1429-1437.

9 Giannelli V, Di Gregorio V, Iebba V, et al: Microbiota and the gut-liver axis: bacterial translocation, inflammation and infection in cirrhosis. World J Gastroenterol 2014:20:16795-16810.

10 Pan C, Gu Y, Zhang W, et al: Dynamic changes of lipopolysaccharide levels in different phases of acute on chronic hepatitis B liver failure. PLoS One 2012;7: e49460.

11 Hartmann P, Seebauer CT, Schnabl B: Alcoholic liver disease: the gut microbiome and liver cross talk. Alcohol Clin Exp Res 2015;39:763-775.
Claria J, Stauber RE, Coenraad MJ, et al: Systemic inflammation in decompensated cirrhosis: characterization and role in acute-on-chronic liver failure. Hepatology 2016;64:1249-1264.

3 Moreau R, Jalan R, Gines P, et al: Acute-on-chronic liver failure is a distinct syndrome that develops in patients with acute decompensation of cirrhosis. Gastroenterology 2013;144:1426-1437, 1437.e1-9.

14 Sarin SK, Kedarisetty CK, Abbas Z, et al: Acute-onchronic liver failure: consensus recommendations of the Asian Pacific Association for the Study of the Liver (APASL) 2014. Hepatol Int 2014;8:453-471.

15 Bernsmeier C, Pop OT, Singanayagam A, et al: Patients with acute-on-chronic liver failure have increased numbers of regulatory immune cells expressing the receptor tyrosine kinase MERTK. Gastroenterology 2015;148:603-615.e14.

16 Jenne CN, Kubes P: Immune surveillance by the liver. Nat Immunol 2013;14:996-1006.

17 Fullerton JN, Gilroy DW: Resolution of inflammation: a new therapeutic frontier. Nat Rev Drug Discov 2016; 15:551-567.

18 Liu H, Ma Z, Lee SS: Contribution of nitric oxide to the pathogenesis of cirrhotic cardiomyopathy in bile ductligated rats. Gastroenterology 2000;118:937-944.

19 Yang YY, Liu H, Nam SW, et al: Mechanisms of TNFalpha-induced cardiac dysfunction in cholestatic bile duct-ligated mice: interaction between TNFalpha and endocannabinoids. J Hepatol 2010;53:298-306.

20 Odeh M: Pathogenesis of hepatic encephalopathy: the tumour necrosis factor-alpha theory. Eur J Clin Invest 2007;37:291-304.

21 Zemtsova I, Gorg B, Keitel V, et al: Microglia activation in hepatic encephalopathy in rats and humans. Hepatology 2011;54:204-215.

22 Chastre A, Belanger M, Nguyen BN, et al: Lipopolysaccharide precipitates hepatic encephalopathy and increases blood-brain barrier permeability in mice with acute liver failure. Liver Int 2014;34:353-361.
23 Durand F, Graupera I, Gines P, et al: Pathogenesis of hepatorenal syndrome: implications for therapy. Am J Kidney Dis 2016;67:318-328.

24 Navasa M, Follo A, Filella X, et al: Tumor necrosis factor and interleukin- 6 in spontaneous bacterial peritonitis in cirrhosis: relationship with the development of renal impairment and mortality. Hepatology 1998;27: 1227-1232.

25 Gustot T: Multiple organ failure in sepsis: prognosis and role of systemic inflammatory response. Curr Opin Crit Care 2011;17:153-159.

26 Fernandez J, Acevedo J, Wiest R, et al: Bacterial and fungal infections in acute-on-chronic liver failure: prevalence, characteristics and impact on prognosis. Gut 2017;DOI: 10.1136/gutjnl-2017-314240.

27 Mücke MM, Rumyantseva T, Mücke VT, et al: Bacterial infection-triggered acute-on-chronic liver failure is associated with increased mortality. Liver Int 2018;38: 645-653.

28 Engelmann C, Berg T: Management of infectious complications associated with acute-on-chronic liver failure. Visc Med 2018;34:DOI: 10.1159/000491107.

29 Remmler J, Schneider C, Treuner-Kaueroff T, et al: Increased level of interleukin 6 associates with increased 90-day and 1-year mortality in patients with end-stage liver disease. Clin Gastroenterol Hepatol 2018;16:730737.

30 Gronbaek H, Rodgaard-Hansen S, Aagaard NK, et al: Macrophage activation markers predict mortality in patients with liver cirrhosis without or with acute-onchronic liver failure (ACLF). J Hepatol 2016;64:813822.

31 Waidmann O, Brunner F, Herrmann E, et al: Macrophage activation is a prognostic parameter for variceal bleeding and overall survival in patients with liver cirrhosis. J Hepatol 2013;58:956-961.

32 Macdonald S, Andreola F, Bachtiger P, et al: Cell death markers in patients with cirrhosis and acute decompensation. Hepatology 2018;67:989-1002. 
33 Michelena J, Altamirano J, Abraldes JG, et al: Systemic inflammatory response and serum lipopolysaccharide levels predict multiple organ failure and death in alcoholic hepatitis. Hepatology 2015;62:762-772.

34 Thursz MR, Richardson P, Allison M, et al: Prednisolone or pentoxifylline for alcoholic hepatitis. N Engl J Med 2015;372:1619-1628.

35 Boetticher NC, Peine CJ, Kwo P, et al: A randomized, double-blinded, placebo-controlled multicenter trial of etanercept in the treatment of alcoholic hepatitis. Gastroenterology 2008;135:1953-1960.

36 Bajaj JS: Review article: potential mechanisms of action of rifaximin in the management of hepatic encephalopathy and other complications of cirrhosis. Aliment Pharmacol Ther 2016;43(suppl 1):11-26.

37 Moreau R, Elkrief L, Bureau C, et al: A randomized trial of 6-month norfloxacin therapy in patients with ChildPugh class C cirrhosis. J Hepatol 2017;66(suppl): S1.

38 Garg V, Garg H, Khan A, et al: Granulocyte colonystimulating factor mobilizes $\mathrm{CD} 34^{+}$cells and improves survival of patients with acute-on-chronic liver failure. Gastroenterology 2012;142:505-512.e1.

39 Duan XZ, Liu FF, Tong JJ, et al: Granulocyte-colony stimulating factor therapy improves survival in patients with hepatitis B virus-associated acute-on-chronic liver failure. World J Gastroenterol 2013;19:1104-1110.
40 Arroyo V, Claria J: Acute-on-chronic liver failure, human serum albumin and immune modulation: the beginning of an exciting adventure. Clin Gastroenterol Hepatol 2018;16:633-636.

41 O’Brien AJ, Fullerton JN, Massey KA, et al: Immunosuppression in acutely decompensated cirrhosis is mediated by prostaglandin E2. Nat Med 2014;20:518-523.

42 Caraceni P, Riggio O, Angeli P, et al: Long-term albumin administration improves survival in patients with decompensated cirrhosis: final results of the ANSWER study. J Hepatol 2017;66(suppl):S93.

43 Guevara M, Terra C, Nazar A, et al: Albumin for bacterial infections other than spontaneous bacterial peritonitis in cirrhosis. A randomized, controlled study. J Hepatol 2012;57:759-765.

44 Thevenot T, Bureau C, Oberti F, et al: Effect of albumin in cirrhotic patients with infection other than spontaneous bacterial peritonitis. A randomized trial. J Hepatol 2015;62:822-830.

45 Pan CX, Tang J, Wang XY, et al: Role of interleukin-22 in liver diseases. Inflamm Res 2014;63:519-525.

46 Kleinschmidt D, Giannou AD, McGee HM, et al: A protective function of IL-22BP in ischemia reperfusion and acetaminophen-induced liver injury. J Immunol 2017;199:4078-4090.
47 Reiberger T, Ferlitsch A, Payer BA, et al: Non-selective betablocker therapy decreases intestinal permeability and serum levels of LBP and IL-6 in patients with cirrhosis. J Hepatol 2013;58:911-921.

48 Villa E, Camma C, Marietta M, et al: Enoxaparin prevents portal vein thrombosis and liver decompensation in patients with advanced cirrhosis. Gastroenterology 2012;143:1253-1260.e1-4.

49 Ubeda M, Lario M, Munoz L, et al: Obeticholic acid reduces bacterial translocation and inhibits intestinal inflammation in cirrhotic rats. J Hepatol 2016;64 1049-1057.

50 Gerth HU, Pohlen M, Pavenstadt H, et al: Extracorporeal liver support of liver failure (Article in German). Z Gastroenterol 2017;55:383-393.

51 Trebicka J, Reiberger T, Laleman W: Gut-liver-axis links portal hypertension to acute-on-chronic liver failure. Visc Med 2018;34:DOI: 10.1159/000490262.

52 Drolz A, Ferlitsch A, Fuhrmann V: Management of coagulopathy during bleeding and invasive procedures in patients with liver failure. Visc Med 2018;34:DOI: $10.1159 / 000491106$. 